\title{
Tratamento endodôntico de pré-molar superior com três raízes: relato de caso
}

\author{
Endodontic treatment of upper premolar with three roots: case report \\ Tratamiento endodóntico de premolar superior con tres raíces: relato de caso
}

Cesar Augusto Perini Rosas

ORCID: https://orcid.org/0000-0002-2234-5531

Universidade Estadual do Norte do Paraná, Brasil

E-mail: cesarperini66@hotmail.com

Rodrigo Zuccolotto Ferraz Caselli

ORCID: https://orcid.org/0000-0002-6089-6502

Faculdade São Leopoldo Mandic, Brasil

E-mail: rodrigocaselli@hotmail.com

Emílio Henrique Rocha Gonçalves Ferreira

ORCID: https://orcid.org/0000-0002-6858-0772

Faculdade São Leopoldo Mandic, Brasil

E-mail: emiliohenriquerocha@gmail.com

Ana Grasiela da Silva Limoeiro

ORCID: https://orcid.org/0000-0003-4633-720X

Faculdade de Ilhéus, Brasil

E-mail: grasielalimoeiro@gmail.com

Járcio Victorino Baldi

ORCID: https://orcid.org/0000-0003-4893-8279

Universidade Estadual do Norte do Paraná, Brasil

E-mail: jarcio.baldi@uenp.edu.br

Rina Andrea Pelegrine

ORCID: https://orcid.org/0000-0003-4175-2121

Faculdade São Leopoldo Mandic, Brasil

E-mail: rinapelegrine@terra.com.br

Carlos Eduardo Fontana

ORCID: https://orcid.org/0000-0002-2868-6839 Pontifícia Universidade Católica de Campinas, Brasil

E-mail: ceffontana@hotmail.com

Carlos Eduardo da Silveira Bueno

ORCID: https://orcid.org/0000-0002-2675-0884

Faculdade São Leopoldo Mandic, Brasil

E-mail: carlosesbueno@terra.com.br

\begin{abstract}
Resumo
O sucesso do tratamento endodôntico, depende do conhecimento da anatomia dental interna. O presente artigo relata um caso raro e o tratamento de um primeiro pré-molar superior apresentando três raízes separadas. Paciente do gênero feminino, 32 anos de idade, foi encaminhada para tratamento endodôntico do primeiro pré-molar superior direito. Ao exame clínico, o dente \#14 não apresentava resposta após o teste de vitalidade e no exame radiográfico revelaram a presença de três raízes. Paciente foi submetida a tratamento endodôntico. O conhecimento da anatomia dental, assim como as possíveis variações é de suma importância para um clinico geral, através de uma radiografia de diagnostico ter uma noção do dente a tratar.
\end{abstract}

Palavras-chave: Anatomia; Endodontia; Dente pré-molar.

\begin{abstract}
The success of endodontic treatment depends on knowledge of the internal dental anatomy. This article reports a rare case and the treatment of a maxillary first premolar with three separate roots. A 32-year-old female patient was referred for endodontic treatment of the upper right first premolar. On clinical examination, tooth \#14 did not respond after the vitality test and radiographic examination revealed the presence of three roots. Patient underwent endodontic treatment. The knowledge of dental anatomy, as well as the possible variations, is of paramount importance for a general practitioner, through a diagnostic radiograph to have an idea of the tooth to be treated.
\end{abstract}

Keywords: Anatomy; Endodontics; Bicuspid.

\section{Resumen}

El éxito del tratamiento de endodoncia depende del conocimiento de la anatomía dental interna. Este artículo reporta un caso raro y el tratamiento de un primer premolar maxilar con tres raíces separadas. Una paciente de 32 años fue 
remitida para tratamiento endodóntico del primer premolar superior derecho. En el examen clínico, el diente \# 14 no respondió después de la prueba de vitalidad y el examen radiográfico reveló la presencia de tres raíces. El paciente se sometió a un tratamiento de endodoncia. El conocimiento de la anatomía dental, así como las posibles variaciones, es de suma importancia para que un médico general, a través de una radiografía diagnóstica, tenga una idea del diente a tratar.

Palabras clave: Anatomia; Endodoncia; Diente premolar.

\section{Introdução}

O sucesso do tratamento do sistema de canais radiculares requer um conhecimento profundo da morfologia da raiz e do canal radicular. Os primeiros pré-molares superiores de três raízes são incomuns (0,5-1\%) e são semelhantes aos dos molares superiores, e são referidos como “pequenos molares” (Praveen et al., 2015). Essas condições anatômicas podem representar um desafio para a terapia pulpar. Mesmo com todos esses cuidados, 23,04\% dos dentes tratados endodonticamente podem resultar em insucesso, devido a não localização de canais radiculares, que podem ser deixados sem tratamento e, consequentemente, com infecção remanescente (Karabucak et al., 2016). A associação de erros técnicos endodônticos e lesões periapicais em pré-molares foi relatada na faixa de 28,9\% a 100\% (Nascimento et al., 2018). Segundo Martins et al. (2019) uma revisão sistemática analisando os primeiros pré-molares superiores, através de tomografia computadorizada, verificou que três raízes encontram em 0,2 a 2,6\%. Essa baixa prevalência representa uma condição pouco comum, o que normalmente presenciamos são duas raízes, com dois canais localizados abaixo das cúspides vestibulares e palatinas (Bulut et al., 2015; Ahmad \& Alenezi 2016). A morfologia interna e externa desses dentes foi investigada usando diferentes técnicas. Trabalho tornando os dentes translúcidos por Pécora et al. (1992) e o estudo de 400 primeiros pré-molares superiores descalcificados que foram injetados com corante por Vertucci e Gegauff (1979), relatam uma incidência de três raízes em 2,5 e 5\%, respectivamente. Uma avaliação de imagem radiográfica periapical criteriosa e a exploração endodôntica podem ajudar na detecção de canais extras e se ainda na dúvida, solicitar uma imagem tomográfica 3D com field of view (FOV) pequeno e alta resolução. O objetivo desse relato foi descrever o caso de um primeiro pré-molar superior com três raízes e aumentar a conscientização dos clínicos gerais sobre a importância da anatomia dental e a relevância da radiografia inicial de diagnóstico.

\section{Metodologia}

O estudo foi aprovado pelo Comitê de Ética e Pesquisa da Faculdade São Leopoldo Mandic, com número do parecer: 4.600.706. Paciente concordou com o tratamento e assinou o Termo de Consentimento Livre e Esclarecido.

Paciente gênero feminino, 32 anos de idade, relata que "durante meses só sentia dor no rosto quando apertava e que essa dor foi descendo até aparecer o abscesso na gengiva” (sic). Procurou um dentista, que fez a urgência abrindo o dente e receitou medicação sistêmica. Retornando ao profissional, o mesmo colocou uma medicação no canal e restaurou provisoriamente, mas a noite começou a doer muito. Paciente relata que removeu o curativo. Lembra que alguns meses atrás, fez uma restauração que tinha uma cárie bem profunda.

Avaliação clínica mostrou que o dente \#14 apresentava restaurações e era sensível à percussão e palpação. A condição periodontal estava dentro da normalidade e o dente \#14 não respondeu aos testes ao frio com o Endo Ice (Maquira, Maringá, Paraná, Brasil). A avaliação radiográfica mostrou a possível presença de três raízes (duas vestibulares e uma palatina) e já tinha sido feita a abertura com acesso aos canais (Figura 1A e 1B). O diagnóstico foi de necrose pulpar com periodontite apical crônica. 
Figura 1. Radiografia periapical ortorradial. Figura 1B. Radiografia periapical distorradial.

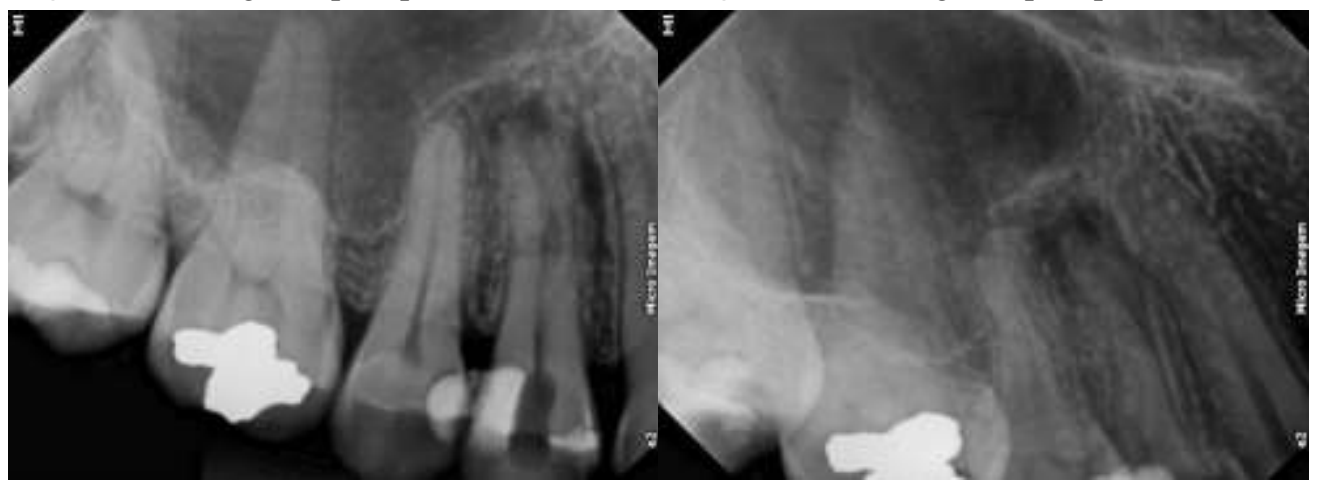

Fonte: Prontuário do paciente.

Foi proposto o tratamento endodôntico a paciente, a qual concordou com ele. O tratamento foi iniciado por aplicação de anestésico tópico (Benzotop 200mg/g, Rio de Janeiro, Brasil), seguido de aplicação de anestesia local com Lidocaína 2\% + 1:100.000 de epinefrina (Alphacaine 100, DFL, Rio de Janeiro, Brasil). Após anestesiado, o dente foi isolado com lençol de borracha e toda câmara pulpar foi copiosamente inundada com hipoclorito de sódio ( $\mathrm{NaOCl}$ ) 2,5\% (Licor de Labarraque, Efficácia, Jacarezinho, Paraná, Brasil) para neutralizar todo conteúdo tóxico. Em seguida, os canais foram explorados manualmente com limas c-pilot \#10 (VDW, Joinville, Santa Catarina, Brasil) até $02 \mathrm{~mm}$ do comprimento aparente do dente. A instrumentação do terço cervical e médio dos canais mesio-vestibular (mv), disto-vestibular (dv) e palatino (p) foi realizado com lima reciprocante X1-Blue \#25.06 (MKLife, Porto Alegre, Rio Grande do Sul, Brasil) acoplado ao motor E Connect Pro (MKLife, Porto Alegre, Rio Grande do Sul, Brasil). Após ampliação cervical, foi determinado o comprimento de trabalho (CT), realizando a patência com lima c-pilot \#10 e utilizando localizador eletrônico foraminal (Propex II, Dentsply/Maillefer, Ballaigues, Suiça) no comprimento apex. Em seguida, os três canais foram instrumentados mecanicamente até o CT (Figura 2) com farta irrigação de $\mathrm{NaOCl}$ 2,5\% e patência feita com lima c-pilot \#10.

Figura 2. Imagem dos canais, após instrumentação mecanizada.

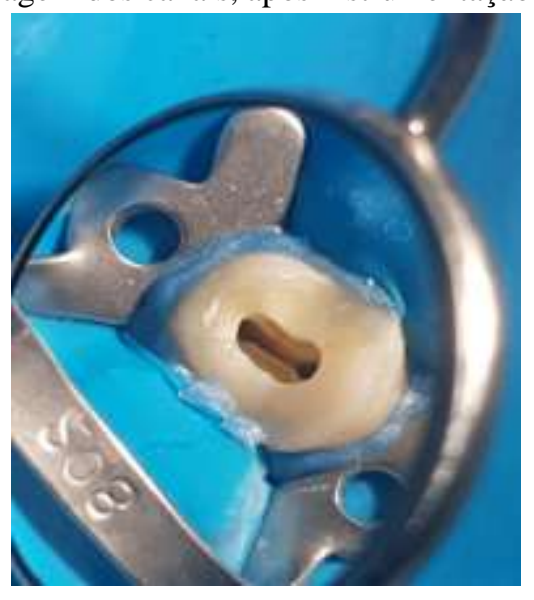

Fonte: Autoria própria.

Após o preparo químico-mecânico, utilizamos ácido cítrico 10\% (Efficácia, Jacarezinho, Paraná, Brasil) em três ciclos de 20 segundos alternando com $\mathrm{NaOCl}$ 2,5\% em três ciclos de 20 segundos para remoção do "smear layer", sendo ativado com ultrassom, colocando um inserto fino E1 irrisonic (Helse Ultrasonic, Santa Rosa de Viterbo, São Paulo, Brasil) a 2 mm do CT. Então os canais foram secos com ponta de papel absorvente (MKLife, Porto Alegre, Rio Grande do Sul, Brasil) e foi realizada 
a prova do cone nos canais dv e p. Após o exame radiográfico (Figura 3), os canais foram obturados pela técnica do cone único com cimento à base de óxido de zinco e eugenol (Endomethasone N, Septodont, França).

Figura 3. Imagem radiográfica da prova do cone dos canais dv e p.

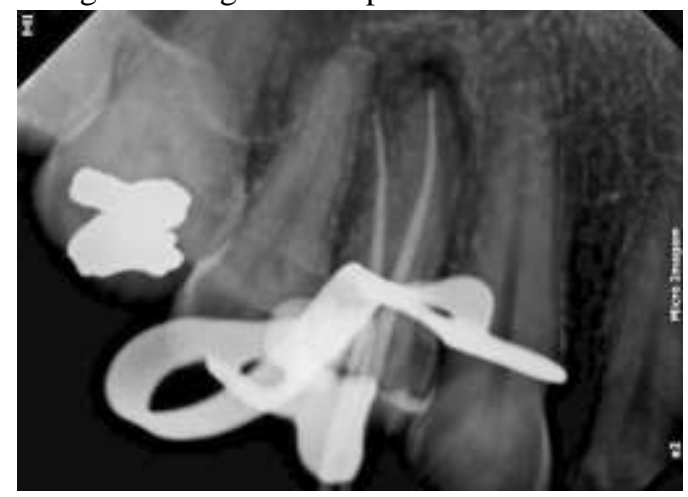

Fonte: Prontuário do paciente

O mesmo procedimento foi realizado no canal mv (Figura 4). Feita a limpeza da câmara pulpar, colocamos um cimento coltosol (Coltene, Vigodent, Bonsucesso, Rio de Janeiro, Brasil) na entrada dos canais e restauramos com material à base de ionômero de vidro (Ionofast, Biodinâmica, Ibiporã, Paraná, Brasil) (Figura 5). Ao final, o dente \#14 foi radiografado, demonstrando completo preenchimento dos três canais radiculares (Figura 6). Paciente retornou após 1 ano e 5 meses para controle radiográfico, onde observou formação óssea na região periapical (Figura 7).

Figura 4. Imagem radiográfica mostrando a obturação dos três canais.

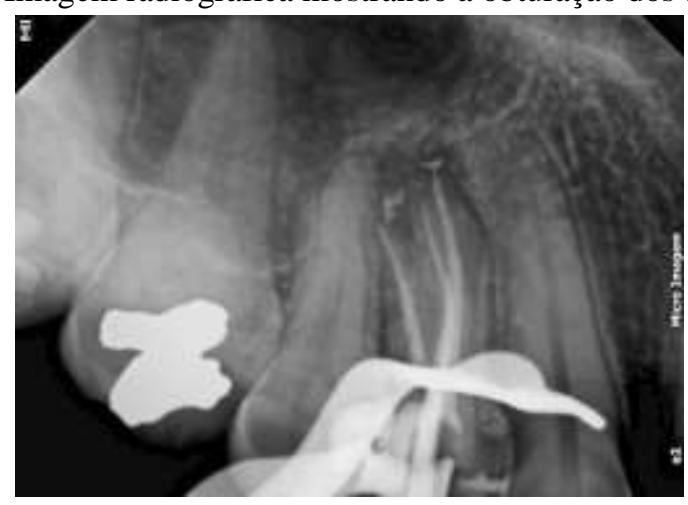

Fonte: Prontuário do paciente. 
Figura 5. Blindagem do dente com material restaurador.

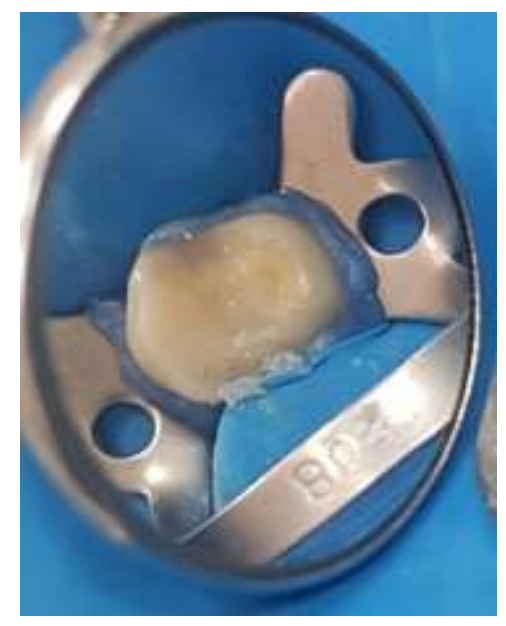

Fonte: Autoria própria.

Figura 6. Radiografia final.

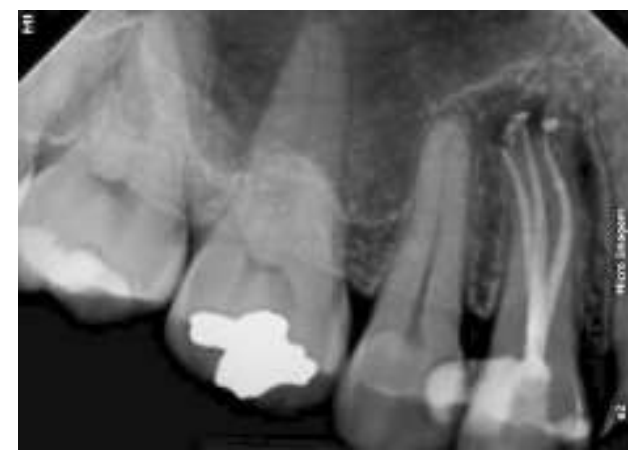

Fonte: Prontuário do paciente.

Figura 7. Radiografia de conrole após 1 ano e 5 meses.

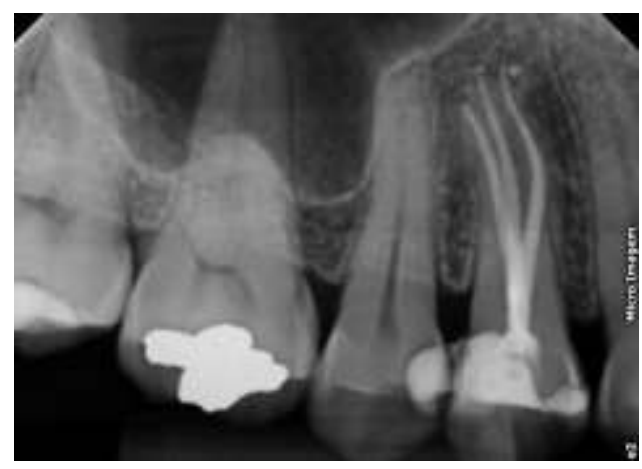

Fonte: Prontuário do paciente.

\section{Discussão}

Este caso mostrou um primeiro pré-molar superior com três canais e três raízes separadas. O conhecimento da morfologia do sistema de canais radiculares e a interpretação cuidadosa das radiografias pré-operatórias é necessário para o sucesso do tratamento endodôntico (Arisu \& Alacam 2009). Segundo Martins et al. (2017), relata que os primeiros pré-molares superiores apresentam três raízes em 2,2\% e que a configuração dos canais radiculares tipo VIII (3-3) de Vertucci com um n= 690, encontraram $5(0,7 \%)$, estudo feito através de tomografia. De acordo com muitas referências na literatura, não há consenso, mas sabemos que três raízes e três canais nos primeiros pré-molares são muito raro (Nimigean et al., 2013). 
Apesar da baixa incidência de três canais, não podemos ignorar. O primeiro pré-molar superior é um dos dentes mais difícil de serem tratados endodonticamente, devido ao número de raízes e número de canais, a direção e frequentes depressões longitudinais das raízes, as várias configurações da cavidade pulpar e as dificuldades em visualizar o limite apical (Pécora et al., 1992).

As imagens radiográficas e a própria análise radiográfica nem sempre são dados suficientes para obter uma abstração adequada do sistema de canais radiculares. A forma e configurações do sistema de canal é sempre variável (Oporto et al., 2013). A tomografia não deve ser usada para avaliação de doença periapical antes do tratamento endodôntico. No entanto, pode ser indicado para auxiliar no diagnóstico de dor (não) odontogênico quando o exame clínico e a avaliação radiográfica convencional não são claros. A tomografia é útil para o arsenal do endodontista para identificar canais radiculares. Para fins endodônticos, o field of view (FOV) deve ser limitado à região de interesse, ou seja, o FOV deve abranger o dente sob investigação e suas estruturas adjacentes (Patel et al., 2015; Cohenca \& Shemesh 2015; Liang \& Yue 2019; Durack \& Patel 2012; Tyndall \& Kohltfarber 2012).

Segundo Mota de Almeida et al. (2014), após os pacientes serem submetidos à tomografia, seguindo as indicações da Comissão Européia, os planos de tratamentos endodônticos foram alterados em 53\% dos casos. Em outro estudo, a imagem de tomografia pré-operatória fornece informações adicionais quando comparadas às radiografias periapicais pré-operatórias, o que pode levar a modificações no plano de tratamento em aproximadamente 62\% dos casos (Jonathan et al., 2014; Patel et al., 2012; Venskutonis et al., 2014).

Uma análise da anatomia dentária na radiografia inicial, bem como uma atenção especial às características da anatomia externa, representa requisitos essenciais para garantir a correta identificação de canais extranumerários (Soares \& Leonardo 2003), embora as anomalias do canal radicular dos primeiros pré-molares superiores apresentarem baixa prevalência, elas devem ser detectadas por avaliação cuidadosa para evitar possíveis falhas endodônticas (Casadei et al., 2020).

No presente caso, uma radiografia com incidência ortorradial e distorradial, mostrou se suficiente para sugerir uma anatomia distinta no primeiro pré-molar superior apresentando com três raízes separadas, mas a tomografia computadorizada de feixe cônico ou cone beam (TCCB) pode ser utilizada como recurso auxiliar na Endodontia, sendo favorável ao tratamento comparada à radiografia periapical por permitir avaliar tecidos duros da região maxilofacial e estruturas tridimensionais em casos complexos, além da possibilidade de visualização corte a corte, possibilitando uma avaliação precisa e melhor planejamento do tratamento pelo profissional (Machado et al., 2021). Treinamento prévio e conhecimento adequado sobre anatomia dental, favoreceram no sucesso do tratamento endodôntico do primeiro pré-molar superior com três canais e três raízes separadas.

\section{Considerações Finais}

O presente estudo teve como objetivo conscientizar os clínicos sobre o conhecimento da anatomia dental, assim como as possíveis variações e da importância do exame clínico e de imagem no diagnóstico.

\section{Referências}

Ahmad, I. A., \& Alenezi, M. A. (2016). Root and root canal morphology of maxillary first premolars: a literature review and clinical considerations. Journal of endodontics, 42(6), 861-872.

Arisu, H. D., \& Alacam, T. (2009). Diagnosis and treatment of three-rooted maxillary premolars. European journal of dentistry, 3(01), 62-66.

Bulut, D. G., Kose, E., Ozcan, G., Sekerci, A. E., Canger, E. M., \& Sisman, Y. (2015). Evaluation of root morphology and root canal configuration of premolars in the Turkish individuals using cone beam computed tomography. European Journal of Dentistry, 9(04), $551-557$. 
Shemesh, H., \& Cohenca, N. (2015). Clinical applications of cone beam computed tomography in endodontics: a comprehensive review. Quintessence Int, 46, 657-668.

Durack, C., \& Patel, S. (2012). Cone beam computed tomography in endodontics. Brazilian dental journal, 23(3), $179-191$.

Ee, J., Fayad, M. I., \& Johnson, B. R. (2014). Comparison of endodontic diagnosis and treatment planning decisions using cone-beam volumetric tomography versus periapical radiography. Journal of endodontics, 40(7), 910-916.

Karabucak, B., Bunes, A., Chehoud, C., Kohli, M. R., \& Setzer, F. (2016). Prevalence of apical periodontitis in endodontically treated premolars and molars with untreated canal: a cone-beam computed tomography study. Journal of endodontics, 42(4), 538-541.

Liang, Y. H., \& Yue, L. (2019). A discussion on three-dimensional digital imaging technology: application of cone-beam CT in endodontics. Zhonghua kou qiang yi хие za zhi= Zhonghua kouqiang yixue zazhi= Chinese journal of stomatology, 54(9), 591-597.

Machado, B. S., Saguchi, A. H., Yamamoto, A. T. A., \& Diniz, M. B. (2021). Uso de tomografia computadorizada no diagnóstico e planejamento endodôntico de pré-molar superior com dupla curvatura radicular. Research, Society and Development, 10(12), e488101220668.

Martins, J. N., Marques, D., Mata, A., \& Caramês, J. (2017). Root and root canal morphology of the permanent dentition in a Caucasian population: a cone-beam computed tomography study. International Endodontic Journal, 50(11), 1013-1026.

Martins, J. N., Marques, D., Silva, E. J. N. L., Caramês, J., \& Versiani, M. A. (2019). Prevalence studies on root canal anatomy using cone-beam computed tomographic imaging: a systematic review. Journal of endodontics, 45(4), 372-386.

Mota de Almeida, F. J., Knutsson, K., \& Flygare, L. (2014). The effect of cone beam CT (CBCT) on therapeutic decision-making in endodontics. Dentomaxillofacial Radiology, 43(4), 20130137.

Nascimento, E., Gaêta-Araujo, H., Andrade, M., \& Freitas, D. Q. (2018). Prevalence of technical errors and periapical lesions in a sample of endodontically treated teeth: a CBCT analysis. Clinical oral investigations, 22(7), 2495-2503.

Nimigean, V., Nimigean, V. R., Sălăvăstru, D. I., \& Buțincu, L. (2013). A rare morphological variant of the first maxillary premolar: A case report. Rom J Morphol Embryol, 54(4), 1173-5.

Oporto, V. G. H., Saavedra, R., Soto, P. C. C., \& Fuentes, R. (2013). Double root anatomical variations in a single patient: endodontic treatment and rehabilitation of a three-rooted first premolar. Case report. Int J Morphol, 31(4).

Patel, S., Brown, J., Pimentel, T., Kelly, R. D., Abella, F., \& Durack, C. (2019). Cone beam computed tomography in endodontics-a review of the literature. International endodontic journal, 52(8), 1138-1152.

Patel, S., Wilson, R., Dawood, A., Foschi, F., \& Mannocci, F. (2012). The detection of periapical pathosis using digital periapical radiography and cone beam computed tomography-Part 2: a 1-year post-treatment follow-up. International endodontic journal, 45(8), 711-723.

Pécora, J. D., Saquy, P. C., Sousa Neto, M. D., \& Woelfel, J. B. (1992). Root form and canal anatomy of maxillary first premolars. Braz Dent J, 2 (2), 87-94.

Praveen, R., Thakur, S., Kirthiga, M., Shankar, S., Nair, V. S., \& Manghani, P. (2015). The radiculous' premolars: case reports of a maxillary and mandibular premolar with three canals. Journal of natural science, biology, and medicine, 6(2), 442.

Soares, J. A., \& Leonardo, R. T. (2003). Root canal treatment of three-rooted maxillary first and second premolars-a case report. International endodontic journal, 36(10), 705-710.

Tyndall, D. A., \& Kohltfarber, H. (2012). Application of cone beam volumetric tomography in endodontics. Australian dental journal, 57, $72-81$.

Venskutonis, T., Plotino, G., Juodzbalys, G., \& Mickevičienė, L. (2014). The importance of cone-beam computed tomography in the management of endodontic problems: a review of the literature. Journal of endodontics, 40(12), 1895-1901.

Vertucci, F. J., \& Gegauff, A. (1979). Root canal morphology of the maxillary first premolar. Journal of the American Dental Association (1939), 99(2), 194198. 